\title{
Robust decentralized switched controller design for linear continuous-time systems
}

\author{
Vojtech Veselý ${ }^{1}$
}

\begin{abstract}
The paper is devoted to obtain original equivalent subsystem method to design of decentralized controller for linear large scale systems. On the theoretical example a new robust decentralized PID switched controller design procedure is obtained for linear time-varying (gain scheduled plant model) uncertain complex system with decentralized output and input structure. Controller design procedure to decentralized controller design performs on the subsystem level. The designed decentralized switched controller ensures the robust stability of closed-loop complex polytopic system with performance $H_{2}$ quadratic cost function (QSR). The proposed practical examples with ideal or non-ideal switch of switching parameters show the effectiveness of equivalent subsystem approach.
\end{abstract}

K e yw ords: large scale system, equivalent subsystem method, switched system, continuous time system, robust stability, output decentralized feedback, quadratic/multi quadratic stability.

\section{Introduction}

Decentralized controller design procedure has been obtained in frequency and in time domain. In the frequency domain the following approaches are presented: independent design approach [3], sequential design [4], and method of equivalent subsystems approach [5]. Decentralized controller design in time domain started with vector Lyapunov function [2]. Large progress has been made using LMI-BMI approach to design the decentralized controller, see survey paper [6]. Unfortunately, when one in the decentralized controller design procedure the the linear (bilinear) matrix inequalities should used the full complex uncertain plant model is need. This paper is devoted to obtain the new unified robust decentralized gain scheduled (switched) controller design procedure which provided on the subsystem level such that design procedure ensure resulting complex plant stability and $H_{2}$ performance for all polytopic uncertain complex system.

Switched control systems have played an important role in the past decades. Motivation for design such systems comes:

- switched systems have numerous practical applications, - in real world, there are systems that cannot be stabilized by continuous or discrete-time controllers, but a stabilizing switching control can be found.

For stability switched systems the quadratic stability play important role, gives the stability under an arbitrary switching law. Multiple Lyapunov function gives however less conservative results. For stability of nonlinear switched system see [1]. In the references there are huge number for switched controller design to discretetime systems but for continuous-time the number of references is rather small [7-12]. For switched controller design in the continuous time system $[10,13]$ the notion of Dwell-Time (minimal time interval between switching) have been introduced. In switched controller design the dwell-time $T_{d}$ is in the term of $e^{A_{c} T d}$ where $A_{c}$ is the closed-loop system. Term mention above complicates the switched controller design procedure for continuous time systems.

In this paper, the original method of equivalent subsystem approach is developed to design of decentralized robust controller. Design procedure ensures the robust quadratic/multi quadratic stability conditions to closedloop switched complex system with arbitrary (or event driven) switching. In the proposed design procedure there is no need to use the approach of dwell-time. The rate of switching variable changes could be described by the designer, ideal switching with infinite rate or non-ideal switching with constant rate, which opens new possibility for practical realizations. In this paper the authors idea [3] were followed and it was spread to robust decentralized control using equivalent subsystem approach.

After formulation of the problem and obtaining the preliminary results to design the equivalent subsystem model, we give the main results to obtain the equivalent subsystems approach and sufficient robust stability conditions for quadratic/multi quadratic stability approach to design the switched controller. The results, in the form of BMI are demonstrated on three examples for ideal and non-ideal switching.

Hereafter, the following notational conditions will be adopted. Given a symmetric matrix $P=P^{\top} \in \mathcal{R}^{n \times n}$,

\footnotetext{
${ }^{1}$ Institute of Robotics and Cybernetics, Faculty of Electrical Engineering and Information Technology, Slovak University of Technology in Bratislava, Ilkovičova 3, 81219 Bratislava, vojtech.vesely@stuba.sk
} 
the inequality $P>0,(P<0)$ denotes matrix positive (negative) definiteness. $I_{n}, 0_{n}$ denotes the identity (zero) matrix of corresponding dimensions.

\section{Preliminaries and problem formulation}

Let the uncertain LTV complex system is governed by

$$
\begin{aligned}
\sum \sigma: \quad \dot{x}(t) & =A(\theta, \xi) x(t)+B(\theta, \xi) u(t), \\
y(t) & =C x(t) \\
x(0) & =x_{0}
\end{aligned}
$$

where $x(t) \in \mathcal{R}^{n}$ is the state vector, $u(t) \in \mathcal{R}^{m}$ is the control vector, $y(t) \in \mathcal{R}^{l}$ is the output vector of the system to be controlled, $\sigma \in S=\{1,2, \ldots, p\}$ is an arbitrarily switching algorithm. The arbitrary switching algorithm $\sigma$ is a piecewise constant, right continuous function which specifies at each time the index of the active closedloop system $[15,21], p$ is the number of switched modes of linear systems and

$$
\begin{aligned}
& A(\theta, \xi)=A_{0}(\xi)+\sum_{i=1}^{p} A_{i}(\xi) \theta_{i} \\
& B(\theta, \xi)=B_{0}(\xi)+\sum_{i=1}^{p} B_{i}(\xi) \theta_{i}
\end{aligned}
$$

where $\xi \in R^{N}$ are constant or time varying uncertain parameters such that

$$
\begin{aligned}
& \xi \in \Omega_{\xi}:=\left\{\xi_{i} \geq 0, \sum_{i=1}^{N} \xi_{i}=1, \sum_{i=1}^{N} \dot{\xi}_{i}=0, i=1,2, \ldots, N\right\}, \\
& \theta \in \Omega_{\theta}:=\left\{\theta_{j} \in\langle 0,1\rangle, \sum_{j=1}^{p} \theta_{j}=1, \sum_{j=1}^{p} \dot{\theta}_{j}=0, \dot{\theta} \in \Omega_{t}\right\}
\end{aligned}
$$

are switching parameters. Note that for switching systems the stable steady state points of switching parameters $\theta_{i}, i=1,2, \ldots, p$ are equal to 0 or 1 . If the switching parameter $\theta_{i}, i=1,2, \ldots, p$ differs from 0 or 1 , it is moving to one of the stable points with the rate of $\theta_{i}$ change $\dot{\theta}_{i}, i=1,2, \ldots, p$.

In this paper two possibilities were considered for switching parameters $\theta_{j}, j=1,2, \ldots, p$ :

- the rates of variation are infinite -ideal switching. In this case for the $k$-th mode hold $\theta_{k}=1$ and $\theta_{j}=0$, for $j=1,2, \ldots p, j \neq i$,

- the rates of switching parameters variations are finite, non-ideal switching, lets' assume that the system (1) switched from $k$-th to $l$-th mode $k, l=1,2, \ldots, p$, $k \neq l$, holds $\theta_{k}+\theta_{l}=1, \dot{\theta}_{k}+\dot{\theta}_{l}=0, \dot{\theta}_{j}, \theta_{j}=0$, $j=1,2, \ldots, p, j \neq k, l$.

Note, that uncertain system (1) consists of two type of vertices. The first set of vertices are due to the system uncertainties, $N$-vertices and the second one is due to the switching variables $\theta$ with $T=p$ vertices.
If I-part of switched controller is need to design for output feedback, then the plant system states (1) need to be augmented with states equal to integral of output variables. For more detail see [2]. Furthermore assume the system (1) allows to design "I" part of switched controller with static output feedback, that is for augmented plant state holds $x^{\top}=\left[\begin{array}{ll}x_{r}^{\top} & e^{\top}\end{array}\right]$ where $\dot{e}=C x$, state vector $x_{r}$ form the output PD feedback and $e$ ensure integral of output feedback. In the following we assume that matrices in (1) have output and input decentralized structure as follows.

$$
\begin{aligned}
& A_{j i}=\left[\begin{array}{ccc}
A_{11 j i} & \ldots & A_{1 m j i} \\
\ldots & \ldots & \ldots \\
A_{m 1 j i} & \ldots & A_{m m j i}
\end{array}\right] \in R^{n \times n}, \\
& B_{j i}=\operatorname{blockdiag}\left[B_{1 j i} \ldots B_{m j i}\right] \in R^{n \times m}, \\
& C=\operatorname{blockdiag}\left[C_{1} \ldots C_{m}\right] \in R^{l \times n}, \\
& j=1,2, \ldots p ; i=1,2, \ldots, N .
\end{aligned}
$$

The division of above matrices into sub-matrices follows from inherent properties of uncertain plant (1).

The problem studied in this paper is to develop the original design procedure based on the equivalent subsystem approach and using its to design of the robust decentralized switched controller. Design procedure is performed on each subsystem level. The design procedure should guarantee the closed-loop complex system robust stability and optimal value of quadratic cost function.

$J_{h}=\int_{t_{o}}^{\infty}\left(x_{h}^{\top} Q_{h} x_{h}+\dot{x}_{h}^{\top} S x_{h}+u_{h}^{\top} R_{h} u_{h}\right) \mathrm{d} t, \quad h=1,2, \ldots, m$

where matrices $Q_{h}, S_{h}, R_{h}$ are positive definite (semidefinite) and positive definite matrices for $h=1,2, \ldots, m$.

The control algorithm is in the form of PID structure for all subsystems

$$
\begin{aligned}
& u_{h}=k_{p h}(\theta) C_{h} x_{r h}+k_{I h}(\theta) e_{h}+k_{d h}(\theta) C_{h} \dot{x}_{r h}=
\end{aligned}
$$

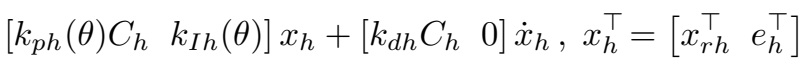

where

$$
k_{x}(\theta)=k_{x o}+\sum_{j=1}^{p} k_{x j} \theta_{j}, \quad \dot{e}_{h}=y_{h} .
$$

$x_{h}$ the $h$-th subsystem state variable including augmented integral output feedback.

The following Lemmas will be useful for the next development.

Definition 1 [22,23]. The switched linear closed-loop subsystem is said to be quadratically stabilizable via output feedback if there exists a Lyapunov function of the form $V_{h}=x_{h}^{\top} P_{h} x_{h}, P_{h}>0$, a positive number $\epsilon>0$ and a switched rule $\sigma$ such that

$$
\frac{\mathrm{d} V_{h}}{\mathrm{~d} t}<-\epsilon x_{h}^{\top} x_{h}
$$


Definition $2[22,23]$. The switched linear closed-loop subsystem is said to be multiple quadratically stabilizable via output feedback if there exists a Lyapunov function of the form $V_{h}(\theta)=x_{h}^{\top} P_{h}(\theta) x_{h}, P_{h}(\theta)>0$, a positive number $\epsilon>0$ and a switched rule $\sigma$ such that

$$
\frac{\mathrm{d} V_{h}(\theta)}{\mathrm{d} t}<-\epsilon x_{h}^{\top} x_{h}
$$

LEMma 1 [4] (Bellman Lyapunov equation).

Consider the uncertain dynamical system (1) with decentralized control structure (4) and control algorithm (6). Decentralized control algorithm (6) belongs to the class of guaranteed cost control law for closed-loop subsystem if and only if there exists decentralized Lyapunov function $V_{h}(x, \theta, \xi), h=1,2, \ldots, m$ and $\varepsilon \geq 0$ such that holds

$$
\begin{aligned}
& B_{e h}=\max _{u}\left\{\frac{d V_{h}(\cdot)}{d t}+J_{h}\left(x_{h}, u_{h}, \dot{x}_{h}\right)\right\}=-\varepsilon x_{h}^{\top} x_{h}, \\
& h=1,2, \ldots, m .
\end{aligned}
$$

Note, that for a particular structure of the Lyapunov function $V_{h}(x, \theta, \xi)$ "if and only if" may be reduced to "if".

Lemma 2. If $D \in R^{n \times n}$ matrix with $\left\{\lambda_{1}, \ldots, \lambda_{n}\right\}$ eigenvalues, then eigenvalues of matrix $D+\alpha I, \alpha \geq 0$ are $\left\{\lambda_{1}+\alpha, \ldots, \lambda_{n}+\alpha\right\}$. Eigenvalues of matrix $(I+c D)$ are $\left\{1+c \lambda_{1}, \ldots, 1+c \lambda_{n}\right\}$.

Lemma 3 [5]. Let $\lambda$ be an eigenvalue of sum of two symmetric matrices $G+D$, then $\lambda \in(F(G)+F(D))$, where $F(\cdot)$ is the field of values of the corresponding matrix.

Lemma 4. The sum of two matrices $G+D$ is stable if and only if there positive definite matrix $P>0$ exists such that the following inequality holds

$$
(G+D)^{\top} P+P(G+D) \leq 0 .
$$

Lemma 5. We are given two stable matrices $A_{1}, A_{2} \in$ $R^{n \times n}$. There are two positive definite matrices $P_{1}>0$, $P_{2}>0$ exist such that

$$
A_{1}^{\top} P_{1}+P_{1} A_{1}+A_{2}^{\top} P_{2}+P_{2} A_{2}<0
$$

then sum of two stable matrices $A_{1}+A_{2}$ is stable if

$$
\left(A_{1}+A_{2}\right)^{\top} P_{1}+P_{1}\left(A_{1}+A_{2}\right)+\left\|A_{2}^{\top} \Delta+\Delta A_{2}\right\| I_{n}<0
$$

where $P_{2}=P_{1}+\Delta$

To ensure that above inequality holds for the sum of matrix $A_{1}+A_{2}$, the exponential degree of stability to matrix $A_{1}$ or $A_{2}$ need to be increased, $[6,7]$.

\section{Main results}

\subsection{Equivalent subsystem approach}

In this section, The Equivalent subsystem design procedure to design of robust decentralized switched controller is obtained. Designed decentralized switched controllers guarantee for the closed-loop uncertain complex system the parameter dependent quadratic stability/ quadratic stability and performance. Above design procedure performs on the subsystem level. Lets' split uncertain polytopic system to the form

$$
\dot{x}=\left(A_{d}(\xi, \theta)+A_{o}(\xi, \theta)\right) x+B(\xi, \theta) u
$$

or for the $i$-th vertex

$$
\begin{aligned}
\dot{x}=\left(A_{d o i}\right. & \left.+\sum_{j=1}^{p} A_{d j i} \theta_{j}+A_{o i}+\sum_{j=1}^{p} A_{o j i} \theta_{j}\right) x \\
& +\left(B_{o j i}+\sum_{j=1}^{p} B_{j i} \theta_{j}\right) u ; \quad i=1,2, \ldots, N .
\end{aligned}
$$

Define, $p_{o i}, i=1,2, \ldots, N$ as

$$
\begin{aligned}
& p_{o i}=\max _{\theta \in \Omega_{\theta}}\left(\max \left(\operatorname{real}\left(\operatorname{eig}\left(A_{o i}+\sum_{j=1}^{p} A_{o j i} \theta_{j}\right)\right)\right)\right) ; \\
& p_{i}=p_{o i}+\delta, \quad \delta>0
\end{aligned}
$$

where $\delta$ is the demanded closed-loop system degree of stability. Lemmna 2 implies, that all eigenvalues of matrix

$$
\begin{aligned}
& \overline{A_{o i}}=A_{o i}+\sum_{j=1}^{p} A_{o j i} \theta_{j}-p_{i} I_{n} \in R^{n \times n} ; \\
& i=1,2, \ldots, N
\end{aligned}
$$

are lying in the left half complex plane, matrix $\bar{A}_{o i}$ is stable. Substituting (11) to (9) one obtains

$$
A_{d o i}+\sum_{j=1}^{p} A_{d j i} \theta_{j}+\overline{A_{o i}}+p_{i} I_{n}=A_{e i}(\theta)+\overline{A_{o i}}
$$

where

$$
\begin{aligned}
A_{e i}(\theta)=A_{d o i} & +\sum_{j=1}^{p} A_{d j i} \theta_{j}+p_{i} I_{n} \\
& =\operatorname{blockdiag}\left\{A_{1 e i}(\theta), \ldots, A_{m e i}(\theta)\right\} \in R^{n \times n}
\end{aligned}
$$

is in the vertex of Equivalent subsystem matrix. Equality (12) implies that if matrix $A_{e i}(\cdot)$ is stable with designed decentralized controller for all $i=1,2, \ldots, N$ than complex system will be stable if the sum of two matrices (12) will be stable for all $i=1,2, \ldots, N$. For $h$-th, $h=1,2, \ldots, m$ closed-loop equivalent subsystem with PID controller one has

$$
\begin{aligned}
& A_{h c i}=A_{h e i}+B_{h i}\left(P I D_{h}\right) \\
& \quad h=1,2, \ldots, m ; i=1,2, \ldots, N .
\end{aligned}
$$


Note, that

$$
\begin{aligned}
A_{c i} & =\operatorname{blockdiag}\left\{A_{1 c i}, \ldots, A_{m c i}\right\}, \\
A_{c} & =\operatorname{blockdiag}\left\{A_{c 1}, A_{c 2}, \ldots, A_{c N}\right\} .
\end{aligned}
$$

Assume, the closed-loop matrices $A_{h c i}, h=1,2, \ldots, m$, $i=1,2, \ldots, N$ are due to designed $P I D_{h}$ switched controller are robust stable for all $\theta \in \Omega_{\theta}$ then the complex system $\bar{A} o+A_{c}$ for adequate choice of $\delta[6,7]$ will be stable. For checking the closed-loop stability of uncertain polytopic system, the multiple Lyapunov function or quadratic stability approach should be used. Complex system is stable if Lyapunov matrix $P_{i}, i=1,2, \ldots, N$ (for quadratic stability if Lyapunov matrix $P$ ) exist such that the following inequality holds

$$
\left(\bar{A}_{o i}+A_{c i}\right)^{\top} P_{i}+P_{i}\left(\bar{A}_{o i}+A_{c i}\right)<0 ; i=1,2, \ldots, N
$$

Theorem 1. Assume, that the $h$-th, $h=1,2, \ldots, m$ decentralized controllers with control algorithm (6) guarantee the closed-loop robust stability and performance for the $h$-th subsystems $A_{h c i}$, that is closed-loop the $i$-th equivalent subsystems $A_{c i}, i=1,2, \ldots, N$ is stable and satisfy inequality (14) or Lemma 5. Designed decentralized robust switched controller guarantees the robust stability with performance of uncertain complex plant (1).

Proof. Inequality (14) imply if $P_{i}>0, i=$ $1,2, \ldots, N$ exists closed-loop equivalent subsystems are stable. From (11) and (12) one obtains

$$
\begin{aligned}
\bar{A}_{o i} & +A_{c i}=A_{o i}+\sum_{j=1}^{p} A_{o j i} \theta_{j}-p_{i} I_{n}+A_{d o i} \\
& +\sum_{j=1}^{p} A_{j i} \theta_{j}+B_{i} \text { PID }+p_{i} \text { In } ; i=1,2, \ldots, N .
\end{aligned}
$$

The sum of two stable matrices $\bar{A}_{o i}+A_{c i}$ is stable if the conditions (14) or Lemma 5. are satisfied which proved that complex uncertain closed-loop system with decentralized switched controller is stable.

From (15) one could observed that for designed static decentralized output/state feedback controller the eigenvalues of closed-loop complex system with equivalent subsystem approach and equivalent interaction and real plant with real interaction are the same, which not may hold (or with small errors) for designed dynamic controllers.

\subsection{Robust decentralized switched controller design using equivalent subsystem approach}

This subsection formulates theoretical approach to obtain decentralized robust PID switched controller design procedure for the uncertain complex plant such a way that switched controller design approach carried out on the subsystem level using in this paper proposed equivalent subsystem approach. The obtained results ensure parameter dependent quadratic stability with multiple
Lyapunov function/guadratic stability - one Lyapunov function to uncertain polytopic closed-loop systems for $\theta \in \Omega_{\theta}, \dot{\theta} \in \Omega_{t}$. The uncertain equivalent subsystem model in the $i$-th vertex is in the form (12)

$$
\dot{x}_{h}=A_{h e i}(\theta)+B_{h i}(\theta) u_{h}, y_{h}=C_{h} x_{h}
$$

where (e-omitted)

$$
\begin{gathered}
A_{h e i}(\theta)=A_{o h e i}+\sum_{j=1}^{p} A_{h e i j} \theta_{j}=A_{o h i}+\sum_{j=1}^{p} A_{h i j} \theta_{j} \\
A_{o h i}=A_{d o i h}+p_{i} I_{h} .
\end{gathered}
$$

Assume, candidate Lyapunov function for the $h$-th subsystem is as follows

$$
\begin{aligned}
& V_{h}\left(x_{h}, \theta, \xi\right)=x_{h}^{\top} P_{h}(\theta, \xi) x_{h}= \\
& x_{h}^{\top}\left(P_{h o}(\xi)+\sum_{j=1}^{p} P_{h j}(\xi) \theta_{j}\right) x_{h} \in R^{n_{h} \times n_{h}}
\end{aligned}
$$

where $P_{h j}(\xi)=\sum_{i=1}^{N} P_{h i j} \xi_{i}, j=0,1,2, \ldots, p, h=$ $1,2, \ldots, m$. The first time derivative of Lyapunov function (17) is given as follows

$$
\begin{aligned}
\frac{\mathrm{d} V_{h}(\cdot)}{\mathrm{d} t} & =v_{h}^{\top}\left[\begin{array}{ccc}
0 & P_{h}(\theta, \xi) & 0 \\
P_{h}(\theta, \xi) & P_{h}(\dot{\theta}, \xi) & 0 \\
0 & 0 & 0
\end{array}\right] v_{h}, \\
v_{h}^{\top} & =\left[\begin{array}{lll}
\dot{x}_{h}^{\top} & x_{h}^{\top} & u_{h}^{\top}
\end{array}\right]
\end{aligned}
$$

where

$$
P_{h}(\dot{\theta}, \xi)=\sum_{i=1}^{N}\left(\sum_{j=1}^{p} P_{h i j} \dot{\theta}_{j}\right) \xi_{i} .
$$

To split Lyapunov matrix $\left(P_{h}(\cdot)\right.$ from the system matrices and ensure convex properties with respect to uncertain variable $\xi$ and switching variable $\theta$ a new auxiliary matrices $N_{i}, i=1,2, \ldots, 6$ with dimensions $N_{i} \in$ $R^{n_{h} \times n_{h}}, \quad i=1,2 ; N_{3} \in R^{n_{h} \times m_{h}} ; N_{i} \in R^{m_{h} \times n_{h}}$, $i=4,5 ; N_{6} \in R^{m_{h} \times m_{h}}$ are introduced as follows

$$
\begin{gathered}
2\left[N_{1} \dot{x}_{h}+N_{2} x_{h}+N_{3} u_{h}\right]^{\top}\left(\dot{x}_{h}-A_{h e i}(\theta)-B_{h i}(\theta) u_{h}\right)=0 \\
=0 \\
2[21) \\
{\left[\begin{array}{ll}
k_{4 h}(\theta) \dot{x}_{h}+N_{5} x_{h}+N_{6} u_{h}
\end{array}\right]^{\top}\left(\dot{x}_{h}\right)=0}
\end{gathered}
$$

Denote $K_{h}(\theta)=\left[\begin{array}{ll}k_{p h}(\theta) C_{h} & k_{I h}\end{array}\right], K_{D h}(\theta)=\left[\begin{array}{ll}k_{d h}(\theta) C_{h} & 0\end{array}\right]$. Summarizing (18), (9) and (20) the time derivative of Lyapunov function to the closed-loop system were obtained for the $h$-th equivalent subsystem and the $i$-th vertex (subscript "e" is omitted)

$$
\frac{\mathrm{d} V_{h i}(\cdot)}{\mathrm{d} t}=v_{h}^{\top} W_{h i} v_{h} \leq 0
$$


where

$$
\begin{aligned}
W_{h i}= & W_{o h i}+\sum_{j=1}^{p} W_{i j h} \theta_{j} \leq 0, \\
W_{o h i}= & \left\{w_{k l h i}^{o}\right\}_{3 \times 3}, \quad W_{i j h}=\left\{w_{k l h i}^{j}\right\}, \\
w_{11 h i}^{o}= & N_{1}^{\top}+N 1-N_{4} K_{d h o}-K_{d h o}^{\top} N_{4}, \\
w_{12 h i}^{o}= & P_{o i}-N_{1}^{\top} A_{h i o}+N_{2}-N_{4}^{\top} K_{h o}-K_{d h o}^{\top} N_{5}, \\
w_{13 h i}^{o}= & -N_{1}^{\top} B_{h i o}+N_{3}+N_{4}^{\top}-K_{d h o}^{\top} N_{6}, \\
w_{22 h i}^{o}= & \sum_{j=1} P_{j i} \dot{\theta}_{j}-N_{2}^{\top} A_{h i o}-A_{h i o}^{\top} N_{2}-N 5^{\top} K_{h o}-K_{h o}^{\top} N_{5}, \\
w_{23 h i}^{o}= & -N_{2}^{\top} B_{h i o}-A_{h i o}^{\top} N_{3}+N_{5}^{\top}-K_{h o}^{\top} N_{6}, \\
w_{33 h i}^{o}= & -N_{3}^{\top} B_{h i o}-B_{h i o}^{\top} N_{3}+N_{6}^{\top}+N_{6}, \\
w_{11 h i}^{j}= & -N^{\top} K_{d h j}-K_{d h j}^{\top} N_{4}, \\
w_{12 h i}^{j}= & P_{j i}-N_{1}^{\top} A_{h i j}-N_{4}^{\top} K_{h j}-K_{d h j}^{\top} N_{5}, \\
w_{13 h i}^{j}= & -N_{1}^{\top} B_{h i j}-K_{d h j}^{\top} N_{6}, \\
w_{22 h i}^{j}= & -N_{2}^{\top} A_{h i j}-A_{h i j}^{\top} N_{2}-N_{5}^{\top} K_{h j}-K_{h j}^{\top} N_{5}, \\
w_{23 h i}^{j}= & -N_{2}^{\top} B_{h i j}-A_{h i j}^{\top} N_{3}-K_{h j}^{\top} N_{6}, \\
w_{33 h i}^{j}= & -N_{3}^{\top} B_{h i j}-B_{h i j}^{\top} N_{3}, \\
& h=, m_{i} ;=1,2, \ldots, N .
\end{aligned}
$$

Closed-loop system is parameter dependent quadratically stable/ quadratically stable for all $\theta \in \Omega_{\theta}, \dot{\theta} \in \Omega_{t}$ and uncertainty $\xi \in \Omega_{\xi}$ if matrix $W_{h i} \leq 0$ for $h=$ $1,2, \ldots, m, i=1,2, \ldots, N$ To obtain the guaranteed cost control algorithm ( $\mathrm{H}_{2}$-approach), the time derivative of Lyapunov function (21) and quadratic cost function (5) to the Bellman Lyapunov equation (7) need to be substituted

$$
B e_{i h}=v_{h}^{\top}\left(W_{h i}+L_{h}\right) v_{h}=v_{h}^{\top} H_{h i}(\theta) v_{h} \leq 0
$$

where

$$
L_{h}=\left[\begin{array}{ccc}
S_{h} & 0 & 0 \\
0 & Q_{h} & 0 \\
0 & 0 & R_{h}
\end{array}\right], H_{h i}=\left\{h_{k l i j}\right\}_{3 \times 3}
$$

From (23) and (24) it is clear that only three variables are changing by summing three matrices $S_{h}$ with $w_{11 h i}^{o}$, $Q_{h}$ with $w_{22 h i}^{o}$ and $R_{h}$ with $w_{33 h i}^{o}$, that is

$$
\begin{aligned}
& h_{11 h i}=w_{11 h i}^{o}+\sum_{j=1}^{p} w_{11 h i}^{j} \theta_{j}+S_{h}, \\
& h_{12 h i}=w_{12 h i}^{o}+\sum_{j=1}^{p} w_{12 h i}^{j} \theta_{j}, \\
& h_{13 h i}=w_{13 h i}^{o}+\sum_{j=1}^{p} w_{13 h i}^{j} \theta_{j},
\end{aligned}
$$

$$
\begin{aligned}
& h_{22 h i}=w_{22 h i}^{o}+\sum_{j=1}^{p} w_{22 h i}^{j} \theta_{j}+Q_{h}, \\
& h_{23 h i}=w_{23 h i}^{o}+\sum_{j=1}^{p} w_{23 h i}^{j} \theta_{j}, \\
& h_{33 h i}=w_{33 h i}^{o}+\sum_{j-1}^{p} w_{33 h i}^{j} \theta_{j}+R_{h} .
\end{aligned}
$$

Inequality (23) is convex with respect to switched parameter $\theta$ and uncertain parameter $\xi$, therefore (22) holds if and only if (22) is negative definite for all vertices $i=1,2, \ldots, N, h=1,2, \ldots, m$ and $j=1,2, \ldots, p$. Robust decentralized switch controller design procedure is based on the inequality (23).

Let's summarize the obtained robust decentralized controller design procedure, (16)-(22), to the following theorem.

THEOREM 2. The uncertain polytopic complex system (1) is robust parameter dependent quadratically stable/ quadratically stable with guaranteed cost if for the performance matrices $Q_{h}, S_{h}, R_{h}, h=1,2, \ldots, m$ and for the given bounds $\theta \in \Omega_{\theta}, \dot{\theta} \in \Omega_{t}, \xi \in \Omega_{\xi}$ there exist auxiliary matrices $N_{i}, i=1,2, \ldots, 6$, symmetric positive definite matrices $P_{i j}, i=0,1,2, \ldots, N, j=1,2, \ldots, p$ such that $P(\theta, \xi)$ is positive definite and for each subsystem controller gain matrices $k_{p h}(\theta), k_{I h}(\theta)$ and $k_{d h}(\theta)$, $h=1,2, \ldots, m$ such that inequality (22) holds.

P r o o f . Sufficient conditions of above Theorem results from the previous consideration.

Note, that equivalent subsystem method to design of decentralized controller former has been obtained in the frequency domain with sufficient and necessary stability conditions [11]. In this paper, obtained equivalent subsystem method in the time domain is with sufficient stability condition. Both methods ensuring the decentralized controller design procedure on the subsystem level.

$\mathrm{R}$ e m a r k 1 . For ideal switching the switching rate of variation $\theta, \dot{\theta}$ is infinite (positive, negative). In this case only in $w_{22 h i}^{o}$ the variable $P_{i j}=0, j=1,2, \ldots, p$, $i=1,2, \ldots, N$. For non-ideal switching the entries of vector $\dot{\theta}$ are finite numbers.

R e m a r k 2. Robust decentralized controller design procedure could be obtained from (22) if $p=0$ is put.

R e m a r k 3. Robust decentralized gain scheduled controller design procedure could be obtained if one changes the set $\Omega_{\theta}(2)$ to the set $\Omega_{\theta}:=\left\{\theta_{j} \in\left\langle\underline{\theta}_{j} \quad \bar{\theta}_{j}\right\rangle\right.$, $j=1,2, \ldots, p$

In this section the new equivalent subsystem method in time domain and originally design procedure to robust decentralized switched controller design are obtained. The obtained robust switched decentralized controller design procedure guarantee to closed loop uncertain complex plant with multiple Lyapunov function/Lyapunov function parameter dependent quadratic 
stability / quadratic stability and performance for all complex closed loop system.

\section{Examples}

For the purpose of demonstrating the advantages of the equivalent subsystem approach to design of robust decentralized switched controller, on the subsystem level, three example will show the effectiveness of the design procedure. For the first uncertain complex plant, two approaches provided the solution for non-ideal switching parameters with maximal rate of variation $\dot{\theta}_{\max }=10 / \mathrm{s}$ and ideal switching parameters with infinite rate of switching parameters changes. PID (dynamic) robust decentralized output feedback switched controller were designed for the first and second example and PI (static) robust decentralized output feedback switched controller for non-ideal switching were designed for the third example. In the above three examples arbitrarily switching algorithm and $H_{2}$ design procedure defined by (22) will be used. The uncertain polytopic complex switched system has two vertices, fourth order and two subsystems is described as

$$
\begin{aligned}
& \left(A(\theta, \xi), B(\theta, \xi)=\left(A_{1}(\theta), B_{1}(\theta)\right) \xi_{1}+\right. \\
& \left(A_{2}(\theta), B_{2}(\theta)\right) \xi_{2}, X(\theta)=X_{o}+X_{1} \theta_{1}
\end{aligned}
$$

For the first vertex $(i=1)$ of complex system parameters are as follows.

$$
\begin{aligned}
A_{10}= & {\left[\begin{array}{cccc}
-1 & 0.3 & 0.28 & 0.1 \\
0.05 & -0.71 & 0.1 & 0.25 \\
0.12 & 0.05 & -0.65 & 0.31 \\
0.27 & 0.13 & 0.07 & -0.7
\end{array}\right], } \\
A_{11}= & {\left[\begin{array}{cccc}
-0.1 & 0.03 & 0.1 & 0.005 \\
0.002 & -0.2 & 0.02 & 0.025 \\
0.02 & 0.05 & -0.2 & 0.005 \\
0.03 & 0.03 & 0.03 & -0.2
\end{array}\right], } \\
B_{10}^{\top}= & {\left[\begin{array}{cccc}
1 & 0 & 0 & 0 \\
0 & 0 & 1.1 & 0
\end{array}\right], B_{11}^{\top}=\left[\begin{array}{cccc}
0.1 & 0 & 0 & 0 \\
0 & 0 & 0.23 & 0
\end{array}\right], } \\
C_{1}= & {\left[\begin{array}{llll}
1 & 0
\end{array}\right] . }
\end{aligned}
$$

The second vertex, $(i=2)$

$$
\begin{aligned}
A_{20}= & {\left[\begin{array}{cccc}
-1.3 & 0.4 & 0.52 & 0.15 \\
0.07 & -0.85 & 0.2 & 0.35 \\
0.17 & 0.07 & -0.7 & 0.4 \\
0.32 & 0.18 & 0.09 & -0.86
\end{array}\right], } \\
A_{21}= & {\left[\begin{array}{cccc}
-0.23 & 0.1 & 0.1 & 0.003 \\
0.001 & -0.2 & 0.015 & 0.022 \\
0.07 & 0.01 & -0.24 & .1 \\
0.02 & 0.015 & 0.01 & -0.3
\end{array}\right], } \\
B_{20}^{\top}= & {\left[\begin{array}{cccc}
1.2 & 0 & 0 & 0 \\
0 & 0 & 1.3 & 0
\end{array}\right], B_{21}^{\top}=\left[\begin{array}{cccc}
0.1 & 0 & 0 & 0 \\
0 & 0 & 0.3 & 0
\end{array}\right], } \\
C_{2}= & {\left[\begin{array}{ll}
1 & 0
\end{array}\right] . }
\end{aligned}
$$

For the three examples quadratic cost function is used in the form of QSR

$$
\begin{aligned}
J_{h} & =\int_{t_{o}}^{\infty}\left(x_{h}^{\top} Q_{h} x_{h}+\dot{x}_{h}^{\top} S_{h} x_{h}+u_{h}^{\top} R_{h} u_{h}\right) \mathrm{d} t, \\
h & =1,2, \ldots, m .
\end{aligned}
$$

The parameters of positive definite matrices are:

$$
\begin{gathered}
Q_{h}=q_{h} I_{h}, \quad q_{h}=0.01 \\
S_{h}=s_{h} I_{h}, s_{h}=0.01, \quad R_{h}=r_{h} I, r_{h}=1 .
\end{gathered}
$$

All Lyapunov matrices are bounded by $0<P<r_{0} I, r_{0}=$ 1000 and $\delta=0.01,(12)$. For switched variable holds $\theta_{1} \in\langle 0,1\rangle$. For non-ideal switching the rate of variation switching variable is $\dot{\theta}_{1 \max }=10 / \mathrm{s}$. The first and second equivalent subsystem should be obtained from (10), (11), and (12). From (10) and two vertices one obtains $p_{i 1}=$ 0.3814 - the first vertex and for the second one $p_{i 2}=$ 0.5353 .

\subsection{Example: Non-ideal switching algorithm}

Using the $\mathrm{H}_{2}$ performance to robust output feedback PID switched controller design procedure, developed in this paper (22), the following robust decentralized switched controller is obtained for the first and second subsystems. The first subsystem, $h=1$

$$
\begin{aligned}
R_{1}(s)=-4.2203-\frac{3.0513}{s}-1.7455 s \\
\quad+\left\{-8.8051-\frac{2.539}{s}-1.7313 s\right\} \theta_{1}
\end{aligned}
$$

and the second subsystem, $h=2$

$$
\begin{aligned}
R_{2}(s)=-9.4973 & -\frac{9.5308}{s}-3.9991 s \\
+ & \left\{-8.8051-\frac{9.9232}{s}-0.027 s\right\} \theta_{1}
\end{aligned}
$$

Note that the sign $(-1)$ means negative feedback. Designed robust decentralized switched controller ensures for the closed-loop equivalent subsystems the following eigenvalues.

$$
\begin{aligned}
i=1, h & =1, \mathrm{EGq}_{11}=\{-0.913 ;-0.398 \pm 0.0426 i\}, \\
h & =2, \mathrm{EGq}_{12}=\{-0.799 \pm 0.732 i ;-0.322\}, \\
i=2, h & =1, E G q_{21}=\{-1.219 ;-0.2111 ;-0.232\}, \\
h & =2, E G q_{22}=\{-0.736 \pm 0.599 i ;-0.334\} .
\end{aligned}
$$

Eigenvalues of uncertain closed-loop complex plant with two vertices when real subsystems are substitute with equivalent subsystems are as follows:

$$
\begin{aligned}
i=1 ; \mathrm{EGc}= & \{-1.123 \pm 0.7355 i ;-1.493 \\
& -0.6267 \pm 0.095 i ;-0.9262\} \\
i=2 ; \mathrm{EGc}= & \{-2.045 ;-1.1513 ; \pm 0.6474 i \\
& -0.6154 \pm 0.1494 i ;-1.104\}
\end{aligned}
$$


Eigenvalues of uncertain closed-loop complex plant with two vertices and with real subsystems for designed of two PID robust decentralized controller are as follows

$$
\begin{aligned}
& i=1 ; \mathrm{EGl}=\{-0.983 \pm 0.9261 i ;-0.5966 ; \\
& -0.9831 \pm 0.3645 i ;-0.83282\}, \\
& i=2 ; \mathrm{EGl}=\{-0.9509 \pm 0.9313 i ;-1.3368 \text {; } \\
& -7532 \pm 0.1582 i ;-1.1262\} \text {. }
\end{aligned}
$$

Note, that due to (15) for the designed of decentralized dynamic PID controller the closed-loop eigenvalues of complex plant with equivalent subsystems and with real subsystems are slightly different.

\subsection{Example: ideal switching algorithm}

For ideal switching rate of variation switching parameter $\dot{\theta}_{1 \text { max }}$ is equal to infinite. For this case $\theta_{1}$ serve as a switching variable. For the same system, performance and constraints as in Section 4.1 the following robust decentralized controller are obtained as follows. The first subsystem, $h=1$,

$$
\begin{aligned}
R_{1}(s)=-4.4926- & \frac{2.952}{s}-1.7523 s \\
& +\left\{-4.8104-\frac{2.73902}{s}-1.7497 s\right\} \theta_{1}
\end{aligned}
$$

and second one, $h=2$

$$
\begin{aligned}
R_{2}(s)=-8.4269 & -\frac{7.2154}{s}-3.9981 s \\
+ & \left\{-6.9459-\frac{8.2777}{s}-1.5657 s\right\} \theta_{1} .
\end{aligned}
$$

Eigenvalues of closed-loop real complex plant in two vertices with the designed of two PID robust decentralized switch controller are as follows:

$$
\begin{aligned}
& i=1 ; \mathrm{EGl}=\{-0.8719 \pm 0.793 i \\
& -0.6725 \pm 0.045 i ;-1.0771 \pm 0.0248 i\}, \\
& i=2 ; \mathrm{EGl}=\{-0.8436 \pm 0.8059 i ; \\
& -1.6108 ;-0.6702 \pm 0.1606 i ;-1.084\} \text {. }
\end{aligned}
$$

\subsection{Example: design of robust PI decentralized switched} controller, non-ideal switching algorithm

For the case of the same system parameters, performance and constraints as in the first example in this subsection the robust static output feedback PI switched decentralized controllers for non-ideal switching has been designed. For two subsystems the following decentralized controller are obtained:

the first subsystem

$$
R_{1}(s)=-4.2568-\frac{3.0732}{s}+\left\{-4.998-\frac{2.5766}{s}\right\} \theta_{1}
$$

and the second subsystem

$$
R_{2}(s)=-2.4968-\frac{4.7581}{s}+\left\{-2.4995-\frac{4.7493}{s}\right\} \theta_{1}
$$

Due to static output feedback (PI controller) the closedloop eigenvalues for the equivalent subsystems and real ones are the same (15) as follows:

The first vertice

$$
\begin{aligned}
i=1 ; \text { EGo }=\{-4.6111 ;-1 & .6888 \pm \\
- & .5784 \pm 0.0814 i ;-0.9177\} .
\end{aligned}
$$

The second vertice

$$
\begin{aligned}
i=2 ; \text { EGo }=\{-5.8118 ;- & .9536 \pm 1.499 i ; \\
& -.6073 \pm 0.1175 i ;-1.1298\} .
\end{aligned}
$$

Results of robust decentralized controller design on the subsystem level mentioned above prove that closed -loop system of complex plant is stable. For this examples it is not needed to check the closed-loop stability of real plant with designed decentralized controller using (14). The designed decentralized controller ensures the closedloop stability with performance defined by quadratic cost function QSR for all polytopic uncertain complex plant which proves the effectiveness of proposed method. After some modification above design procedure one could obtain the robust decentralized controller design method using another control methods like as $L_{2}$ gain $\left(H_{\infty}\right)$, regional pole placement approaches and so on.

\section{Conclusion}

This paper is devoted to obtain the original equivalent subsystem method in time domain to design of decentralized controller and a new design procedure to design of the robust decentralized switched controller on subsystem level. Consequently the advantages of the proposed method can be summarized as follows:

- the method of equivalent subsystem approach is developed to design of decentralized controller on subsystem level,that is the order of design model is equal to order of corresponding subsystem,

- the robust switched decentralized controller is designed on the subsystem level such that the stability of closed-loop complex system is guaranteed,

- less conservative results with respect to using dwelltime approach can be obtained for the continuous time system,

- for the switched decentralized robust controller design there is no need to use for continuous time system the approach of "dwell-time" which markedly complicates the design procedure,

- the rate of the switching variable can be prescribed by the designer (actually above value defined the value of 
dwell-time), which opens the new possibilities for practical realizations and development of new theoretical approaches,

- the obtained design procedure to design of robust decentralized switched controller for output/state feedback ensures the closed loop parameter dependent quadratic stability/ quadratic stability of switched systems and guaranteed cost,

- the obtained robust decentralized design procedure can be easily implemented to the standard LMI or BMI approaches,

- the obtained design procedure can be easily transformed to the case of robust gain scheduled decentralized controller design, robust decentralized controller design for continuous-time uncertain polytopic system.

Numerical examples illustrate the effectiveness of the proposed approach.

\section{Acknowledgments}

The work has been supported by the Slovak Scientific Grant Agency, Grant VEGA No. 1/0754/19

\section{REFERENCES}

[1] M. Mattias and D. Liberzon, "Input /output to state stability of switched nonlinear systems", ACC, pp. 1708-1712, June 2010.

[2] V. Vesely and D. Rosinova, "Robust PID-PSD controller design. BMI approach", Asian Journal of Control, vol. 15, no. 2, pp. 469-478, 2013.

[3] V. Vesely and A. Ilka, "Novel approach to switched controller design for linear continuous -time systems", Asian journal of control, vol. 18, no. 4, pp. 1365-1375, 2016.

[4] V. M. Kuncevich and M. M. Lycak, "Control system design using Lyapunov function approach", Nauka, Moscow, 1977 (in Russian).

[5] H. Wielandt, "On the eigenvalues of $\mathrm{A}+\mathrm{B}$ and $\mathrm{AB}$, Journal of research of the National Burrea of Standarts", Mathematical Sciences, vol. 77B, no. 1-2, pp. 61-63, 1973.

[6] D. D. Siljak, Large-scale Dynamic Systems, Stability and Structure, North-Holland, NewYork, 1978.

[7] V. Vesely, "Large-scale dynamic system stabilization using the principle of dominant subsystem approach", Kybernetika, vol. 24, no. 1, pp. 48-61, 1993.

[8] V. M. Matrosov, "On the theory of stability motion", Prikladnaja matematika i Mekhanika, no. 26, pp. 992-1002, 1962.

[9] M. Hovd and S. Skogestad, "Improved independent design of robust decentralized control", Journal of process Control, vol. 3, no. 1, pp. 43-51, 1993.

[10] M. Hovd and S. Skogestad, "Sequantial design of decentralized controllers", Automatica, vol. 30, no. 10, pp. 1601-1607, 1994.
[11] A. Kozakova, V. Vesely, and Kucera, "Robust Decentralized Controller Design Based on Equivalent Subsystems, Automatica", 107, pp. 29-35, 2019.

[12] L. Bakule, "Decentralized Control: An Overview", Annual Reviews Control, vol. 32, pp. 87-98, 2008.

[13] D. Jabri, D. Eddine, Ch. Belkhiat, K. Guelton and . Manomanmi, "Decentralized Controller Design for Large Scale Switched Takagi-Sugeno Systems with $H_{\infty}$ performance specification", Journal od advanced Engineering and Computation, vol. 12, no. 2, pp. 139-150, 2018.

[14] D. Liberzon, "Switching Systems and Control", Systems \& Control: Foundations \& Applications, Birkhäuser Boston, 2003.

[15] J. Lunze and F. Lamnabhi-Lagarrigue, "', Handbook of Hybrid Systems Control: Theory, Tools, Applications, Cambridge University Press, 2009.

[16] J. Geromel and P. Colaneri, "Stability and Stabilization of Continuous-Time Switched Linear Systems", SIAM Journal on Control and Optimization, vol. 45, no. 5, pp. 1915-1930, 2006.

[17] R. Guo and Y. Wang, "Stability analysis for a class of switched linear systems", Asian Journal of control, vol. 14, no. 3, pp. 817-826, 2012.

[18] L. Allerh and U. Shaked, "Robust Stability and Stabilization of Linear Switched Systems With Dwell Time", IEEE Transactions on Automatic Control vol. 56, no. 2, pp. 381-386, 2011.

[19] J. Lygeros, "An Overview of Research Areas Hybrid Control", 44th IEEE Conference on Decision and Control, 2005 and 2005 European Control Conference. CDC-ECC, pp. 5600-5605, 2005.

[20] M. Cao and A. S. Morse, "Dwell-time switching", Systems and Control Letters, vol. 59, pp. 57-65, 2010

[21] M. Matthias, A. Muller and . Liberzon, "Input/output-to state stability of switched nonlinear systems", ACC, pp. 1708-1712, 2010.

[22] V. Veselý and D. Rosinová, "Roobust MPC Controller Design for Switched Systems Using Multi-Parameter Dependent Lyapunov Function", International Journal of Innovative Computing, Information and Control (IJICIC) vol. 10, no. 1, pp. 269-280, 2014.

23] M. Branicky, "Multiple Lyapunov functions and other analysis tools for switched and hybrid systems", IEEE Transactions on Automatic Control vol. 43, no. 4, pp. 475-482, 1998.

Received 17 June 2020

Vojtech Veselý was born in Velké Kapušany, Slovakia in 1940. He received MSc degree in Electrical Engineering from the Leningrad Electrical Engineering Institute, St. Peterburg, Russia, in 1964, PhD and DSc degrees from the Slovak University of Technology, Bratislava, Slovakia, in 1971 and 1985, respectively. Since 1964 he has been with the Department of Automatic Control Systems, STU FEI in Bratislava. Since 1986 he has been a full professor. His research interests include the areas of power system control, decentralized control of large-scale systems, robust control, predictive control and optimization. He is author or coauthor of more than 300 scientific papers.

Received 17 June 2020 